\title{
Detection of ovarian cancer ( \pm neo-adjuvant chemotherapy effects) via ATR-FTIR spectroscopy: comparative analysis of blood and urine biofluids in a large patient cohort
}

\author{
Panagiotis Giamougiannis ${ }^{1,2} \cdot$ Camilo L. M. Morais ${ }^{2} \cdot$ Brice Rodriguez $^{1}$ • \\ Nicholas J. Wood ${ }^{1} \cdot$ Pierre L. Martin-Hirsch ${ }^{1} \cdot$ Francis L. Martin ${ }^{3}$ \\ Received: 29 April 2021 / Revised: 6 June 2021 / Accepted: 9 June 2021 / Published online: 1 July 2021 \\ (C) The Author(s) 2021
}

\begin{abstract}
Ovarian cancer remains the most lethal gynaecological malignancy, as its timely detection at early stages remains elusive. Attenuated total reflection Fourier-transform infrared (ATR-FTIR) spectroscopy of biofluids has been previously applied in pilot studies for ovarian cancer diagnosis, with promising results. Herein, these initial findings were further investigated by application of ATR-FTIR spectroscopy in a large patient cohort. Spectra were obtained by measurements of blood plasma and serum, as well as urine, from 116 patients with ovarian cancer and 307 patients with benign gynaecological conditions. A preliminary chemometric analysis revealed significant spectral differences in ovarian cancer patients without previous chemotherapy $(n=71)$ and those who had received neo-adjuvant chemotherapy-NACT $(n=45)$, so these groups were compared separately with benign controls. Classification algorithms with blind predictive model validation demonstrated that serum was the best biofluid, achieving $76 \%$ sensitivity and $98 \%$ specificity for ovarian cancer detection, whereas urine exhibited poor performance. A drop in sensitivities for the NACT ovarian cancer group in plasma and serum indicates the potential of ATR-FTIR spectroscopy to identify chemotherapy-related spectral changes. Comparisons of regression coefficient plots for identification of biomarkers suggest that glycoproteins (such as CA125) are the main classifiers for ovarian cancer detection and responsible for smaller differences in spectra between NACT patients and benign controls. This study confirms the capacity of biofluids' ATR-FTIR spectroscopy (mainly blood serum) to diagnose ovarian cancer with high accuracy and demonstrates its potential in monitoring response to chemotherapy, which is reported for the first time.
\end{abstract}

Keywords Ovarian cancer $\cdot$ Chemotherapy $\cdot$ Biofluids $\cdot$ Liquid biopsies $\cdot$ ATR-FTIR spectroscopy $\cdot$ Spectroscopy

\section{Introduction}

Ovarian cancer is the 8th most common cancer-related cause of death in women internationally, with incidence and mortality projected to increase markedly in the next 20 years [1,2]. It also represents the most fatal gynaecological cancer due to nonspecific symptoms at its presentation, leading in most cases $(>$

Francis L. Martin

flm13@biocel.uk

1 Department of Obstetrics and Gynaecology, Lancashire Teaching Hospitals NHS Foundation Trust, Preston PR2 9HT, UK

2 School of Pharmacy and Biomedical Sciences, University of Central Lancashire, Preston PR1 2HE, UK

3 Biocel Ltd, Hull HU10 7TS, UK
$70 \%$ ) to being diagnosed in advanced stages of disease [3]. Epithelial ovarian cancer (EOC) represents the most common type (approximately $90 \%$ of cases), with histological subclassifications including serous, mucinous, endometrioid, and clear-cell adenocarcinomas and carcinosarcomas. Among these, highgrade serous carcinomas compose the largest group, comprising $70-80 \%$ of all EOCs. Other rarer histologies include stromal and germ cell tumours [3, 4]. Current treatment modalities include a combination of surgery and chemotherapy, with the most commonly used chemotherapy agents being platins (either cisplatin or carboplatin) with the addition of a taxane (paclitaxel). In cases where upfront operating is deemed unlikely to achieve complete cytoreduction, chemotherapy is initiated first (in regimes described as neo-adjuvant chemotherapy-NACT) followed by interval debulking surgery (IDS) and subsequent administration of further chemotherapy cycles $[4,5]$. 
In an attempt to increase earlier detection of ovarian cancer, large trials have been conducted, which used a combination of imaging (pelvic ultrasonography) and serum biomarkers [cancer antigen CA125, human epididymis protein 4 (HE4)] to produce screening or diagnostic algorithms [6-9]. However, their outcomes failed to demonstrate robust reduction in mortality rates. Additionally, CA125, which is the most commonly used serum biomarker, lacks adequate sensitivity and specificity as it can be raised in many other non-gynaecological malignancies and non-malignant conditions (such as benign ovarian cysts, uterine fibroids, adenomyosis, and endometriosis) $[10,11]$. Particularly in endometriosis, its levels can be significantly elevated in moderate or severe disease [12]. Other tests used in ovarian cancer diagnosis (such as computed tomography, magnetic resonance imaging, tissue biopsies) are expensive, invasive, and time consuming.

The lack of currently available 'gold standards' in ovarian cancer detection has led to an urgent need for the development of new accurate, minimally invasive and cost-effective diagnostic tools. One of the promising new modalities is vibrational spectroscopy, which has shown great potential in the classification between normal and pathological biological materials [13]. Attenuated total reflection Fourier-transform infrared (ATR-FTIR) spectroscopy is a frequently used vibrational spectroscopic technique, which has been experimentally applied in human tissues and biofluids [14]. Its main advantage compared to other infrared (IR) techniques is that the evanescent wave produced by the IR source penetrates the sample only by a few microns, rendering it particularly convenient for assessment of biofluids. ATR-FTIR spectra represent a series of wavenumber absorbance intensities produced by molecular motion upon interaction with the IR source $[15,16]$. Additionally, the "bio-fingerprint" region in the mid-IR area of the electromagnetic spectrum contains absorbing frequencies for many biomolecules, which can lead to the discovery of new biomarkers [17].

Regarding biological substrates, blood and urine are ideal candidates for cancer screening or diagnosis as they are easy to collect and require minimal (venepuncture) or noninvasive (urine collection) procedures [15]. Blood plasma and serum (which is plasma without clotting factors) contain a multitude of constituents without the presence of erythrocytes, which can interfere with spectra obtained from other important biomolecules, and are considered more suitable biofluids than whole blood for spectroscopic investigations in cancer research [18]. Blood or urine ATR-FTIR spectroscopy has been experimentally applied in several types of cancer including breast [19], brain [20, 21], prostate [22], gastrointestinal [23-25] and endometrial [26, 27]. Its performance has also been explored in ovarian cancer, for investigation of diagnostic accuracy $[18,27]$ and identification of new biomarkers [27, 28], by studies involving small datasets, with promising results.
For the acquisition of reliable and clinically interpretable results, appropriate chemometric techniques are applied. Vibrational spectroscopic data are inherently multivariate and require complex analytical approaches. Such multivariate methods include principal component (PCA) and linear discriminant analysis (LDA), which can achieve segregation between different classes [29]. The diagnostic potential of spectroscopy can be further enhanced by applying machine learning methods, which utilise a wide range of classification algorithms such as linear discriminant classifier (LDC) and support vector machines (SVMs) [30]. Herein, the potential of ATR-FTIR spectroscopy in ovarian cancer diagnosis was further interrogated, through spectrochemical analyses in blood and urine samples from a large patient cohort. Additionally, as part of recruited ovarian cancer patients had received NACT, chemotherapy effects on acquired spectra were also explored.

\section{Materials and methods}

\section{Patients and samples}

Between April 2018 and November 2019, 423 consecutive patients were recruited ( $n=116$ with ovarian cancer, $n=307$ with benign gynaecological conditions as controls). The majority of ovarian cancer patients $(n=71)$ had not received chemotherapy whereas the rest $(n=45)$ had received NACT. For the latter group, the median number of NACT cycles was 4 with a median interval of 3 weeks between cycles; 41 patients received a combination of carboplatin and paclitaxel and 4 patients, single-agent carboplatin. Blood samples were collected from all participants and urine from all patients with ovarian cancer. Three patients from the control group did not provide urine. Informed consent was taken from all participants. Samples were collected upon patients' attendance to Royal Preston Hospital (UK) for surgery and therefore were fasting samples. Serum CA125 levels were measured for all patients at the time of their attendance for surgery and for ovarian cancer patients who received NACT at the time of their disease diagnosis as well. Table 1 contains epidemiological as well as CA125 data for the separate study groups. For NACT ovarian cancer patients, the mean interval between completion of their pre-operative chemotherapy and IDS was 39 days. For each patient, two blood samples were obtained, one in tubes containing EDTA anticoagulant and one in serum gel tubes. Urine was collected following urethral cleansing and sterile urinary catheterisation without the use of lubricant gel. Blood samples were centrifuged at $2200 \mathrm{rpm}$ for $15 \mathrm{~min}$ (local protocol), to obtain plasma and serum from EDTA and serum gel tubes respectively. Plasmas and serums were subsequently snap-frozen in liquid nitrogen and stored at $-80{ }^{\circ} \mathrm{C}$. Urines were also stored at $-80{ }^{\circ} \mathrm{C}$ without centrifugation and snap-freezing. 
Table 1 Epidemiological and serum CA125 data for the different study groups. CA125 level was considered elevated if measuring $>35 \mathrm{u} / \mathrm{ml}$

\begin{tabular}{ll}
\hline & Mean [range] \\
\hline Ovarian cancers & \\
Age & $63[20-84]$ \\
All patients $(n=116)$ & $61[20-84]$ \\
No NACT $(n=71)$ & $65[43-83]$ \\
NACT $(n=45)$ & \\
BMI $\left(\mathrm{kg} / \mathrm{m}^{2}\right)$ & $26.7[16.6-48.6]$ \\
All patients $(n=116)$ & $27.4[18.2-48.6]$ \\
No NACT $(n=71)$ & $25.8[16.6-36.4]$ \\
NACT $(n=45)$ & \\
CA125 $(\mathrm{u} / \mathrm{ml})$ & $590[5-8366]$ \\
Non-chemotherapy group $(n=71)$ & $2595[62-23455]$ \\
Chemotherapy group, before NACT $(n=45)$ & $135[8-1104]$ \\
Chemotherapy group, after NACT $(n=45)$ & \\
Benign controls (n=307) & $47[19-89]$ \\
Age & $28.5[17.3-49.8]$ \\
BMI $\left(\mathrm{kg} / \mathrm{m}^{2}\right)$ & $55[1-2627]$ \\
CA125 $(\mathrm{u} / \mathrm{ml})$ &
\end{tabular}

NACT neo-adjuvant chemotherapy

Prior to slide preparation, samples were thawed at room temperature and $60 \mu \mathrm{l}$ of individual biofluids was pipetted on naked FisherBrand $^{\mathrm{TM}}$ and Leica ${ }^{\mathrm{TM}}$ glass slides. All slides were allocated a specific serial number for patient confidentiality. Following overnight drying, samples were transferred to the laboratory in wooden slide trays for ATR-FTIR spectroscopic analysis. All slides were stored in a de-humidified glass container to prevent sample condensation and physical damage. The study was granted ethical approval by the East of England - Cambridge Central Research Ethics Committee (archival genito-urinary tissue, blood, urine, saliva and ascitic fluid collection; REC reference: 16/EE/0010; IRAS project ID: 195311).

Identification of pathology for all participants, as well as staging for patients with ovarian cancer, was based on histopathology reports after processing of surgical specimens. All ovarian cancer patients had no other synchronous malignancies. Tables 2 and 3 demonstrate staging data for ovarian cancers and histological diagnoses for the whole cohort, respectively. For most ovarian cancers, the histological subtype was epithelial (with $66 \%$ being high-grade serous carcinomas) and only one patient had a germ cell tumour. Staging of ovarian malignancies was conducted according to the International Federation of Gynecology and Obstetrics (FIGO) system [32]. Twenty eight percent of ovarian cancer patients were early stage (FIGO I or II) and all NACT patients had advanced metastatic disease (FIGO III or IV). Further demographic data (including patient comorbidities) are available in non-patient identifiable databases.
Table 2 FIGO staging of ovarian cancer patients

\begin{tabular}{lll}
\hline & $\begin{array}{l}\text { Non- } \\
\text { NACT }\end{array}$ & NACT \\
\hline IA & 10 & - \\
IC & 16 & - \\
IIA & 5 & - \\
IIB & 2 & - \\
IIIA & 5 & - \\
IIIB & 4 & 1 \\
IIIC & 27 & 36 \\
IVA & 2 & 5 \\
IVB & - & 3 \\
\hline
\end{tabular}

NACT neo-adjuvant chemotherapy

\section{Spectral acquisition}

ATR-FTIR spectra were obtained via a Bruker TENSOR 27 FTIR spectrometer with Helios ATR attachment, containing a diamond crystal (Bruker Optics Ltd., Coventry, UK) and operated using OPUS 6.5 software. Spectra for each sample were randomly acquired from ten different points. Data acquisition parameters were as follows: $8 \mathrm{~cm}^{-1}$ spectral resolution giving $4 \mathrm{~cm}^{-1}$ data spacing, 32 scans, $6 \mathrm{~mm}$ aperture setting and $2 \times$ zero-filling factors. The ATR diamond crystal was cleaned with distilled water and dried with tissue paper between different samples. A background absorption spectrum (for atmospheric correction) was taken prior to each new sample analysis.

\section{Computational analysis}

The chemometric techniques applied in this study have been previously described by our group [33]. Only the spectral fingerprint region was used for data analysis. Spectral preprocessing consisted of the following: Savitzky-Golay (SG) smoothing (window of 7 points, 1st-order polynomial fitting) and 2 nd derivative followed by vector normalisation. SG smoothing corrects for random noise, 2nd derivative corrects for baseline distortions and resolves fine spectral structure such as closely aligned peaks (despite potential for reduced signal-to-noise ratio), and vector normalisation corrects for physical differences between samples such as thickness, light scattering and concentrations [34]. In addition, the 10 spectral replicas per sample were averaged in order to work on samplebasis models. Exploratory and discriminant analyses were performed with the pre-processed and mean-centred data. Principal component analysis (PCA) was used for exploratory analysis [35]. With this method, pre-processed spectra are decomposed into a certain number of principal components (PCs), which account for the majority of variance within the dataset. Each PC is composed of scores and loadings; the 
Table 3 Histopathological data for the entire cohort. Staging of endometriosis patients was based on intra-operative findings according to the American Society of Reproductive Medicine staging system [31]

\begin{tabular}{|c|c|c|}
\hline & \multicolumn{2}{|c|}{ No. of patients } \\
\hline & No NACT & NACT \\
\hline \multicolumn{3}{|l|}{ Ovarian cancers } \\
\hline High-grade serous & 33 & 43 \\
\hline Low-grade serous & 5 & - \\
\hline Primary peritoneal serous & - & 1 \\
\hline Mucinous & 10 & - \\
\hline Endometrioid & 9 & - \\
\hline Clear cell & 8 & - \\
\hline Carcinosarcoma & 4 & 1 \\
\hline Anaplastic & 1 & - \\
\hline Immature teratoma & 1 & - \\
\hline \multicolumn{3}{|l|}{ Benign controls } \\
\hline \multicolumn{3}{|l|}{ Ovarian cysts (non-endometriomas) } \\
\hline Cystadenomas, fibromas, cystadenofibromas & 71 & \\
\hline Mature cystic teratomas & 13 & \\
\hline Follicular & 3 & \\
\hline Haemorrhagic & 2 & \\
\hline Struma ovarii & 2 & \\
\hline Brenner & 1 & \\
\hline Sertoli-Leydig & 1 & \\
\hline Indeterminate & 2 & \\
\hline \multicolumn{3}{|l|}{ Endometriosis (including endometriomas) } \\
\hline Stage 1 & 16 & \\
\hline Stage 2 & 9 & \\
\hline Stage 3 & 19 & \\
\hline Stage 4 & 28 & \\
\hline Uterine fibroids and/or adenomyosis & 69 & \\
\hline Pelvic inflammatory disease & 9 & \\
\hline Endometrial/cervical polyps & 8 & \\
\hline Hydrosalpinx/paratubal cysts & 7 & \\
\hline Uterine prolapse & 3 & \\
\hline Peritoneal leiomyomatosis & 1 & \\
\hline Endometrial hyperplasia & 1 & \\
\hline Cervical intraepithelial neoplasia (CIN) & 1 & \\
\hline Normal (no pathology identified) & 41 & \\
\hline
\end{tabular}

NACT neo-adjuvant chemotherapy

former is used to detect clustering patterns, relevant to chemical similarities or dissimilarities among samples, and the latter for identification of spectral bands (wavenumbers), which can separate samples from different biological classes, and therefore can be associated with possible spectral biomarkers.

PCA models were built using the PLS Toolbox version 7.9.3 (Eigenvector Research, Inc., USA), and discriminant analysis was performed using the Classification Toolbox for
MATLAB [36]. Furthermore, partial least squares discriminant analysis (PLS-DA) was also used as a comparative technique. PLS-DA is a feature extraction and classification algorithm, based on a linear model for which the classification criterion is obtained by PLS [37]. In PLS-DA, a PLS model is applied to pre-processed spectra, reducing the original spectral variables to a few latent variables in an iterative process, where the class labels for each sample are known in the training set. Then, a threshold value that divides the classes' regions is found [38].

\section{Statistical analysis}

The classification models were evaluated by calculating some figures of merit (accuracy, sensitivity, specificity and F-score) in the test set, composed of $30 \%$ of samples selected by using the Morais-Lima-Martin (MLM) algorithm [39]. Training samples, composed of $70 \%$ of the dataset, were used for model construction via training and cross-validation [for sample splitting methodology, see Supplementary information (ESM) Table S1]. The accuracy represents the total number of samples correctly classified considering true and false negatives, the sensitivity represents the proportion of positives (i.e. ovarian cancer samples) correctly identified, the specificity represents the proportion of negatives (i.e., benign control samples) correctly identified and the F-score measures the overall model performance considering imbalanced classes [40]. These parameters are calculated as follows:

$$
\begin{gathered}
\text { Accuracy }(\%)=[(\mathrm{TP}+\mathrm{TN}) /(\mathrm{TP}+\mathrm{FP}+\mathrm{TN}+\mathrm{FN})] \times 100 \\
\text { Sensitivity }(\%)=[\mathrm{TP} /(\mathrm{TP}+\mathrm{FN})] \times 100 \\
\text { Specificity }(\%)=[\mathrm{TN} /(\mathrm{TN}+\mathrm{FP})] \times 100 \\
\text { F-score }(\%)=(2 \times \mathrm{SENS} \times \mathrm{SPEC}) /(\mathrm{SENS}+\mathrm{SPEC})
\end{gathered}
$$

where TP stands for true positives, TN for true negatives, FP for false positives and FN for false negatives. SENS stands for sensitivity and SPEC for specificity.

$P$-values were calculated for two-dimensional PCA score plots using a MANOVA test and for individual wavenumbers based on an ANOVA test. Statistical significance was considered at $P<0.05$ and statistical high significance at $P<0.001$.

\section{Results and discussion}

Application of spectroscopy can generate important information about constituents of biological samples. The "fingerprint area" at $1800-900 \mathrm{~cm}^{-1}$ in particular provides crucial data, which can lead to characterisation of several key biomolecules [14]. Herein, ATR-FTIR spectroscopy was used to identify the potential of plasma, serum and urine in a large prospective study for the detection of ovarian cancer. The study groups, comprising ovarian cancers with variable histologies and stages of disease, and benign controls with a wide range of 
gynaecological pathologies, provide a "real-world" clinical setting. Additionally, the consecutive recruitment of participants eliminated the risk of patient selection bias.

An initial exploratory analysis was performed to identify potential significant spectral differences between two subgroups in each of the study's main patient cohorts (Fig. 1). For controls, this comparison was made between patients with endometriosis (a condition that has a propensity towards ovarian malignancy) [41] and patients with all other benign pathologies. PCA scores identified no difference for blood plasma or serum and a marginal statistically significant difference for urine $(P=0.01)$. Therefore, controls were used as one entity in comparisons. On the other hand, the comparison between ovarian cancer patients without previous chemotherapy and post-NACT reveals high statistically significant differences for blood plasma or serum $\left(P \approx 10^{-4}\right)$ and marginal statistically significant difference for urine $(P=0.01)$. As this indicated a potential effect of chemotherapy on spectra, these subgroups were compared separately against the whole cohort of controls.

Exploratory analysis using two-dimensional PCA scores plots suggests that serum is the best for differentiation of both groups of ovarian cancer from benign controls (Fig. 2). PLSDA was the best discrimination algorithm in all comparisons and for all biofluids, as it consistently achieved the highest F- scores (see ESM Table S2). Figure 3 demonstrates discriminant function plots on training and test samples, obtained with this algorithm for the three biofluids. With regard to bloodderived biofluids, serum achieved the highest sensitivity, specificity and accuracy $(76 \%, 98 \%$ and $94 \%$ respectively) in the diagnosis of ovarian cancer. Plasma's statistical metrics were also satisfactory though slightly lower $(71 \%, 84 \%$ and $81 \%$ respectively). A very interesting finding is the drop in sensitivity for identification of ovarian cancer post-NACT in both biofluids ( $57 \%$ for serum and $64 \%$ for plasma), whereas high specificities and accuracies are maintained (85-96\%) (Table 4). Similar trends are observed in the other two classification algorithms (PCA-LDA, SVM) used in this study (see ESM Table S2). This finding could suggest a shift towards a more "benign" pattern in spectra obtained from blood biofluids post chemotherapy and is reported for the first time. It is also consistent with the fact that all NACT ovarian cancer patients had chemo-sensitive disease, exhibiting a substantial drop in CA125 at IDS from baseline levels (Table 1), and a reduction in tumour load at their interval computed tomography scan.

Obtained regression coefficient (RC) plots for identification of key biomarkers support the aforementioned conclusion (Fig. 4). In plasma, there were eight peaks with RC $>1$ for the non-chemotherapy ovarian cancers but only three for the
PLASMA
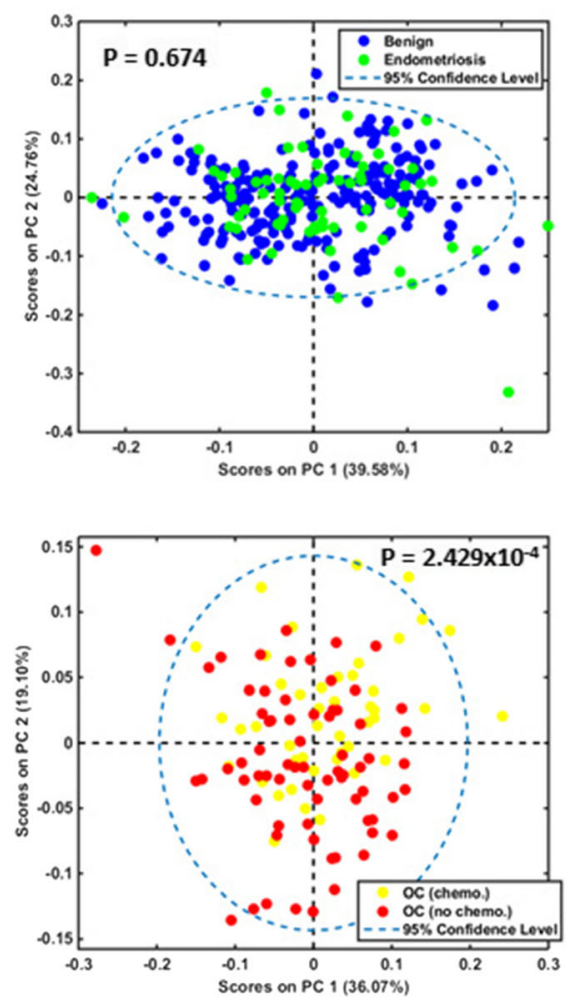

Fig. 1 PCA score plots with $P$-values for intra-class comparisons in plasma, serum and urine. Top graphs: non-endometriosis (benign) versus endometriosis benign controls. Bottom graphs: non-chemotherapy (OC
SERUM
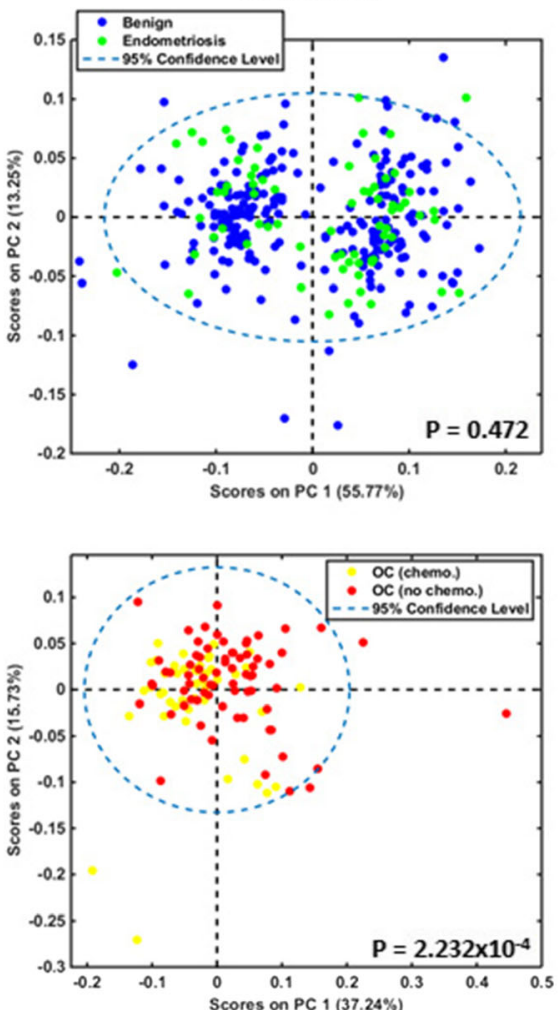

URINE
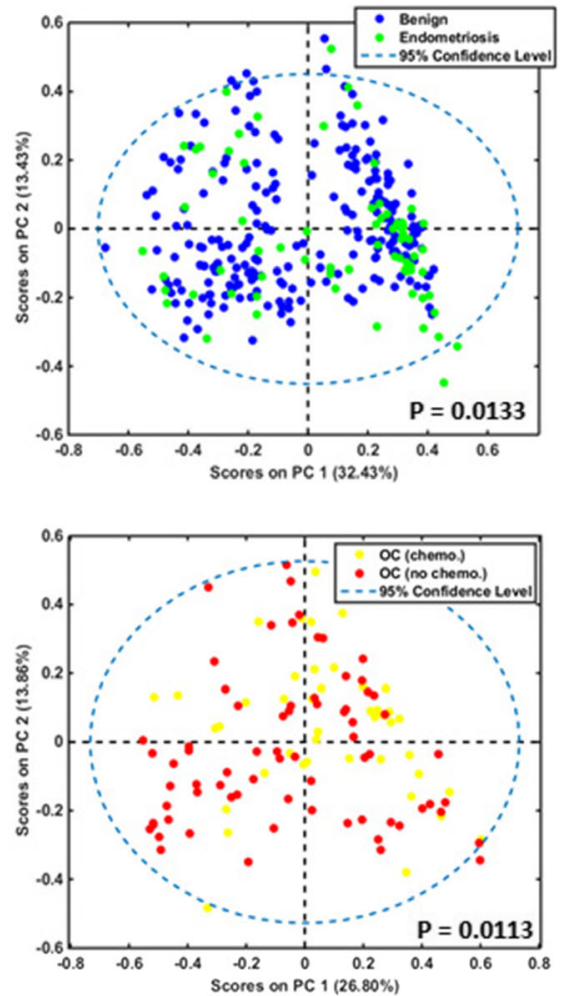

no chemo) versus NACT (OC chemo) ovarian cancer patients. OC, ovarian cancers; chemo, chemotherapy; NACT, neo-adjuvant chemotherapy 
PLASMA
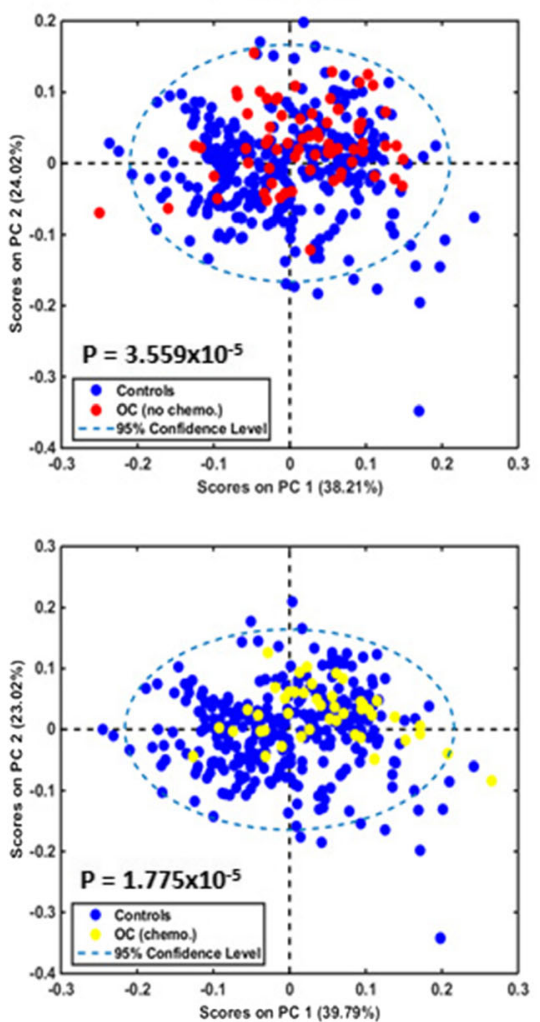

SERUM
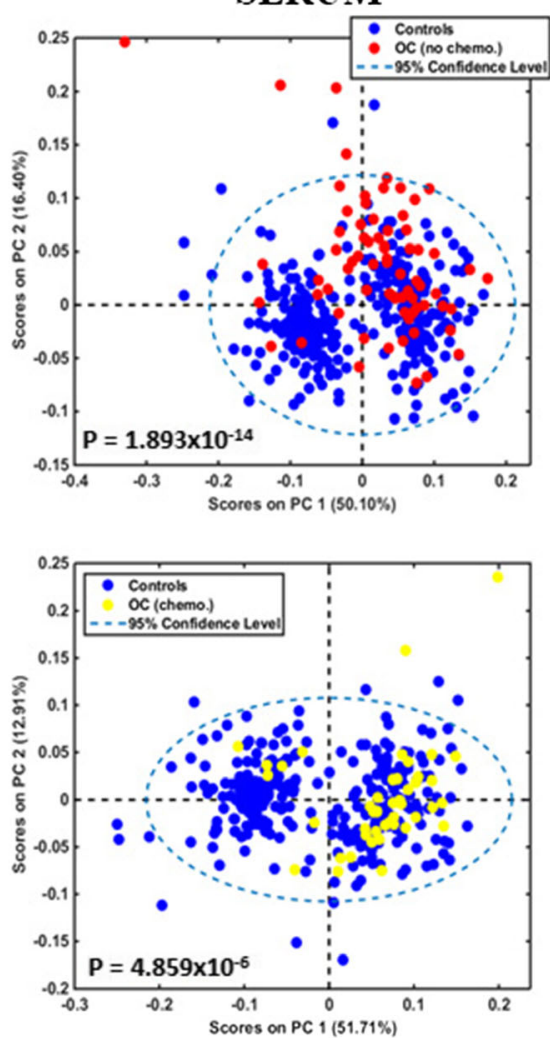

URINE
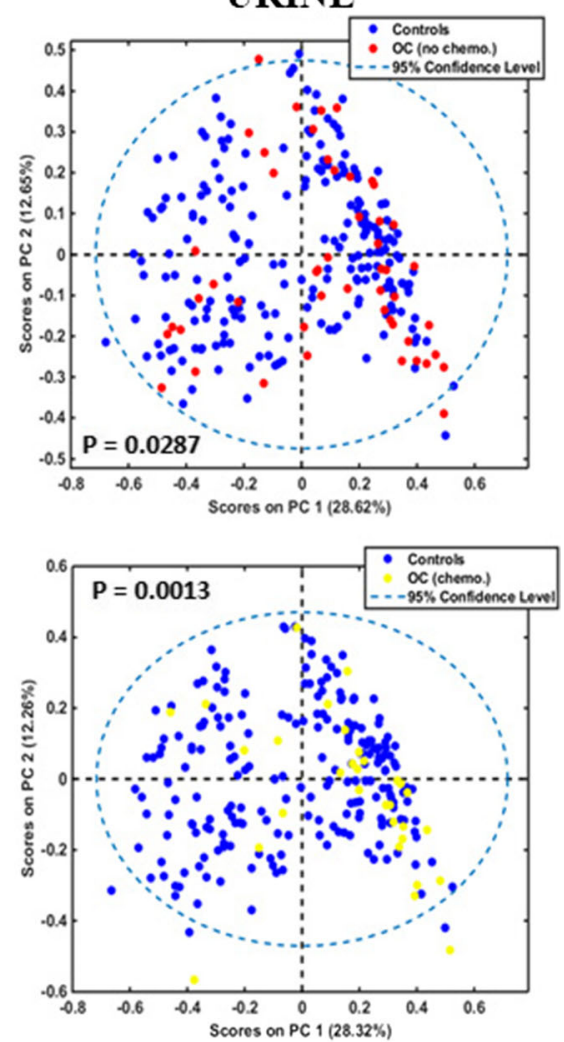

Fig. 2 PCA score plots with $P$-values for inter-class comparisons in plasma, serum and urine. Top graphs: non-chemotherapy ovarian cancers (OC no chemo) versus all benign controls (controls). Bottom graphs:

NACT patients in comparisons with controls. In serum, respective comparisons provided seven peaks with $\mathrm{RC}>4$ for the former group but none with $\mathrm{RC}>2$ for the latter. These observations suggest more "subtle" differences between spectra from post-chemotherapy ovarian cancers and benign patients. Additionally, they probably account for the higher sensitivity, specificity and accuracy obtained with serum in classification of ovarian cancers without previous chemotherapy, as well as the bigger drop in sensitivity post-NACT compared to plasma.

Interestingly, in both biofluids, the majority of peaks are observed at $1000-1200 \mathrm{~cm}^{-1}$ and $1500-1700 \mathrm{~cm}^{-1}$ wavelength regions, which have been assigned to glycoproteins $[42,43]$. Spectral absorbances arise from vibrations of carbohydrates' sugar rings at the former and peptide bonds at the latter [42-44]. Among other glycoproteins that are detected in blood of patients with ovarian cancer and benign gynaecological pathologies (such as HE4, CA15-3, CA724), CA125 has by far the highest molecular weight (due to its heavy glycosylation and lengthier protein backbone) and exhibits the widest concentration differences between ovarian malignancy and non-malignant states [45-48]. It is therefore likely that CA125 is the main glycoprotein contributing to absorbances in the two aforementioned wavelength regions.
NACT ovarian cancers (OC chemo) versus all benign controls (controls). OC, ovarian cancers; chemo, chemotherapy; NACT, neo-adjuvant chemotherapy

CA125 belongs to the big family of mucins, all being glycoproteins, and CA125 is their largest member [49]. Vibrational bands characteristic for mucins have been described at the spectral region $1040-1120 \mathrm{~cm}^{-1}$ [50], at which serum (predominantly) and plasma featured several peaks. Remarkably, absorbance intensities were stronger for the non-chemotherapy ovarian cancers compared to NACT patients in both biofluids, which correlates with the observed magnitude of differences in mean CA125 levels between each of these two groups and controls (Fig. 4, Table 1).

Peaks at RC plots are produced by differences in constituents of biofluids between cases and controls. CA125 has been found to express varied isoforms in ovarian malignancies and benign gynaecological pathologies due to its differential glycosylation in these two entities. In benign conditions, CA125 glycan chains are predicted to contain higher amounts of galactose and $\mathrm{N}$-acetylglucosamine carbohydrates, whereas in ovarian cancer there is a higher content in sialic acid and mannose glycoside residues [51-54]. FTIR spectroscopy has been previously applied for characterisation of carbohydrates $[42,55]$. Prominent peaks for sialic acid and mannose have been identified at approximately $1030 \mathrm{~cm}^{-1}$ and $1070 \mathrm{~cm}^{-1}$, respectively [42], and these peaks had consistently higher absorbance intensities in the ovarian cancer groups compared to 
PLASMA
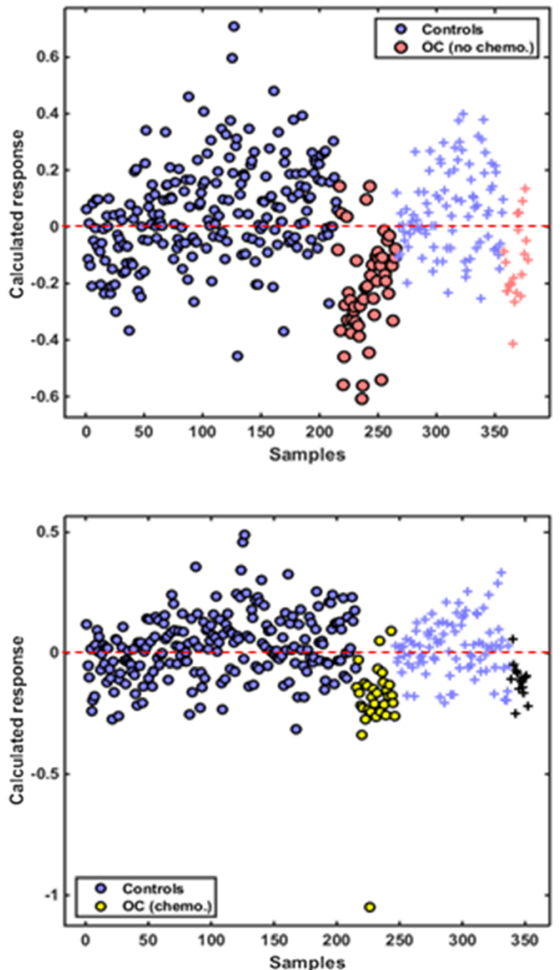

SERUM
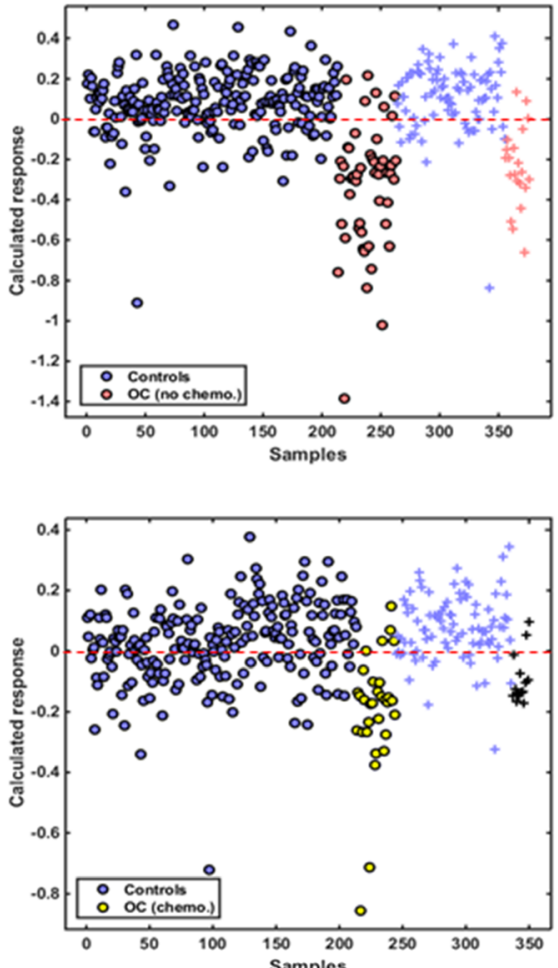

URINE
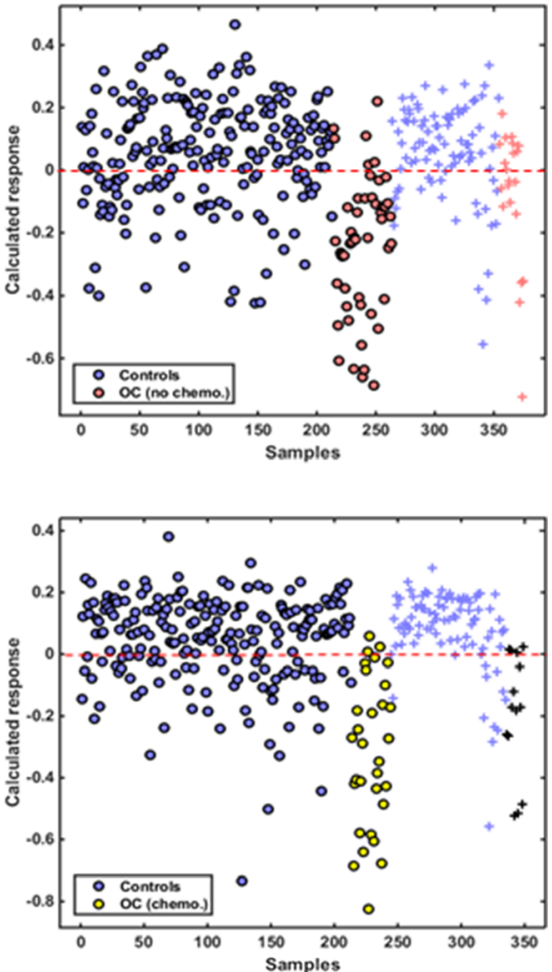

Fig. 3 PLS-DA discriminant function plots for plasma, serum and urine. Top graphs: non-chemotherapy ovarian cancers (OC no chemo) versus all benign controls (controls). Bottom graphs: NACT ovarian cancers (OC

controls. On the other hand, peaks that could be attributed to $\mathrm{N}$-acetylglucosamine and galactose (at approximately $1000 \mathrm{~cm}^{-1}$ and $1160 \mathrm{~cm}^{-1}$, respectively) $[42,55,56]$ exhibited consistently higher absorbance in controls (Fig. 4). These observations could suggest a potential for ATR-FTIR spectroscopy to discriminate between benign and malignant CA125 isoforms. Of note, all the aforementioned peaks had lower RCs in the comparisons of NACT patients versus controls (Fig. 4), implying a potential for spectroscopic identification of chemotherapy effects in ovarian cancer.

A peak that merits attention is observed at $1740 \mathrm{~cm}^{-1}$, assigned to the $\mathrm{C}=\mathrm{O}$ stretching vibration of lipids [57]. This band demonstrated consistently higher RCs in the NACT group compared to non-chemotherapy ovarian cancer patients chemo) versus all benign controls (controls). OC, ovarian cancers; chemo, chemotherapy; NACT, neo-adjuvant chemotherapy. $\mathrm{o}=$ training samples; $+=$ test samples

in plasma and serum (Fig. 4). Interestingly, use of platinum and taxane chemotherapy agents has been associated with hyperlipidaemia in testicular and breast carcinomas respectively $[58,59]$, and increased cellular lipid consumption has been associated with chemoresistance in ovarian cancer [60]. Cancer cells utilise high amounts of lipids to meet their energy demands, and increased circulating lipids following effective chemotherapy might reflect a decrease in this metabolic activity with decelerated tumour growth [59, 60]. As all NACT patients in our study had chemo-sensitive disease, their increased blood lipid levels might be an indirect measure of their response to treatment. Although other factors may have contributed to these changes (such as cachexia in ovarian cancer patients without previous treatment and improved appetite
Table 4 PLS-DA statistical metrics in the classification of the two ovarian cancer groups (nonchemotherapy-OC no chemo, NACT - OC with chemo) from benign controls for plasma, serum and urine

\begin{tabular}{|c|c|c|c|c|c|c|}
\hline & \multicolumn{2}{|l|}{ Plasma } & \multicolumn{2}{|l|}{ Serum } & \multicolumn{2}{|l|}{ Urine } \\
\hline & $\begin{array}{l}\text { OC no } \\
\text { chemo }\end{array}$ & $\begin{array}{l}\text { OC with } \\
\text { chemo }\end{array}$ & $\begin{array}{l}\text { OC no } \\
\text { chemo }\end{array}$ & $\begin{array}{l}\text { OC with } \\
\text { chemo }\end{array}$ & $\begin{array}{l}\text { OC no } \\
\text { chemo }\end{array}$ & $\begin{array}{l}\text { OC with } \\
\text { chemo }\end{array}$ \\
\hline Sensitivity & $71 \%$ & $64 \%$ & $76 \%$ & $57 \%$ & $29 \%$ & $57 \%$ \\
\hline Specificity & $84 \%$ & $88 \%$ & $98 \%$ & $96 \%$ & $87 \%$ & $92 \%$ \\
\hline Accuracy & $81 \%$ & $85 \%$ & $94 \%$ & $91 \%$ & $76 \%$ & $88 \%$ \\
\hline
\end{tabular}

$O C$ ovarian cancers, chemo chemotherapy, NACT neo-adjuvant chemotherapy 
PLASMA
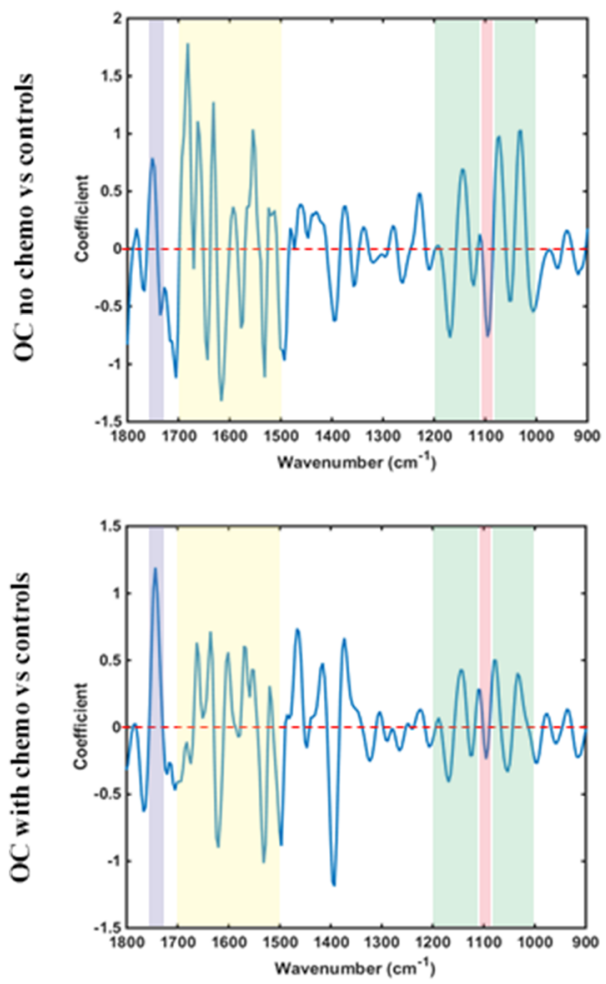

SERUM
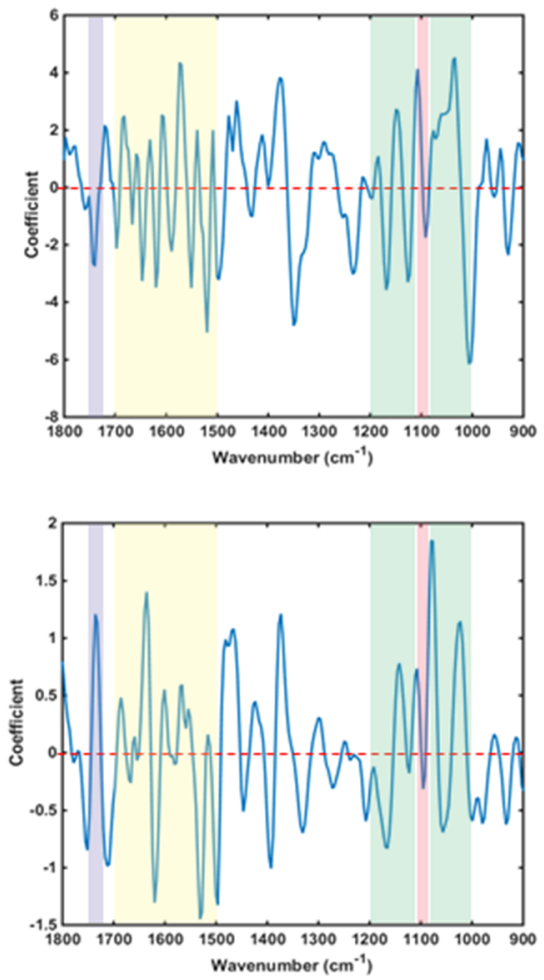

URINE
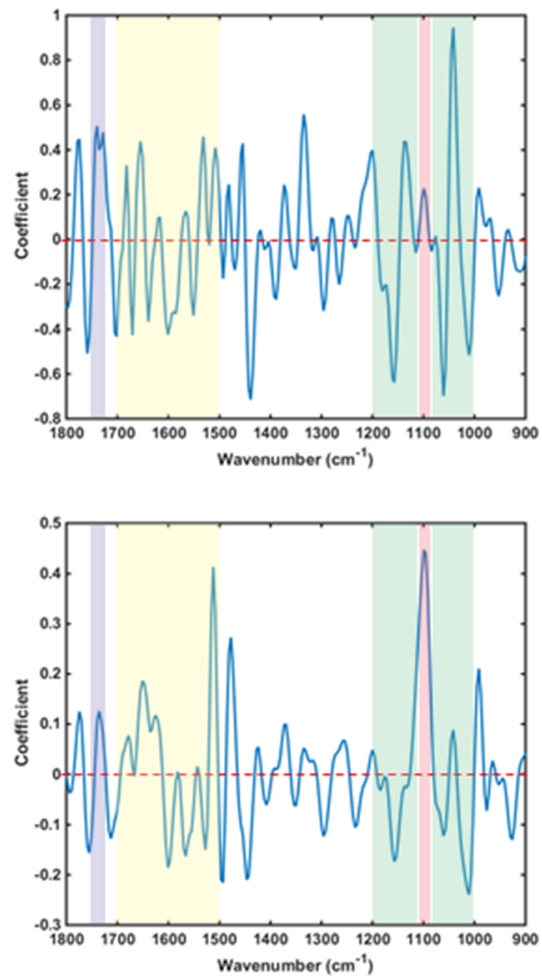

$C=O$ stretching vibration of lipids

Peptide bonds vibrations of glycoproteins
Carbohydrate vibrations of glycoproteins

Phosphate vibration of DNA, polyamines
Fig. 4 PLS-DA regression coefficient plots for identification of spectral biomarkers in plasma, serum and urine. Key wavenumber regions have been marked with different colours. Top graphs: non-chemotherapy ovarian cancers (OC no chemo) versus all benign controls (controls). Bottom

leading to higher lipid intake after NACT), spectral changes at $1740 \mathrm{~cm}^{-1}$ could exhibit potential for monitoring response to chemotherapy.

Contrary to plasma and serum, PCA scores for urine demonstrated marginal statistically significant differences in the comparisons between ovarian cancer groups and controls (Fig. 2). This was reflected in the poor sensitivity (29\%) for diagnosis of ovarian cancer patients without previous chemotherapy obtained with the PLS-DA algorithm. Accuracy was also lower $(76 \%)$ whereas a fairly high specificity was maintained $(87 \%)$. A possible reason for these suboptimal results could be the use of un-centrifuged urine, containing contaminants (such as microorganisms and cellular material) that might have obscured signals from important biomolecules. Strikingly though, sensitivity was two times higher for the NACT ovarian cancer group (57\%), demonstrating an opposite trend to what was observed in plasma and serum. Specificity and accuracy were also improved $(92 \%$ and $88 \%$ respectively) (Table 4). PCA-LDA and SVM algorithms provided similar trends (see ESM Table S2). Additionally, the majority of peaks at $1000-1200 \mathrm{~cm}^{-1}$ and $1500-1700 \mathrm{~cm}^{-1}$ graphs: NACT ovarian cancers (OC chemo) versus all benign controls (controls). OC, ovarian cancers; chemo, chemotherapy; NACT, neoadjuvant chemotherapy

wavelength areas had much smaller RCs $(<0.4)$ and generally exhibited similar amplitudes with peaks in other spectral regions (Fig. 4). This observation suggests that glycoproteins (including CA125) demonstrated much lower absorbance intensities in urine compared to plasma and serum. Indeed, there was only one prominent peak allocated to mucins in the nonchemotherapy ovarian cancer group (at $1040 \mathrm{~cm}^{-1}$ ) [50], which exhibited a substantial drop in NACT patients (from RC 0.9 to 0.1 ) (Fig. 4). These results are probably due to the markedly low urinary CA125 concentrations, with much smaller mean differences between patients with ovarian cancer and benign gynaecological conditions than the ones present in blood [47, 61].

Taking into consideration the aforementioned findings, it is likely that biomolecules other than glycoproteins contributed to the higher sensitivity obtained in urine for the NACT ovarian cancer patients. The band with the highest $\mathrm{RC}$ in this group (and the only one demonstrating a considerable drop in the non-chemotherapy ovarian cancers-from an approximate RC 0.45 to 0.2 ) was located at $1080-1100 \mathrm{~cm}^{-1}$ (Fig. 4). This band has been assigned to phosphate vibrations of 
DNA but also contains a peak at the IR spectrum of polyamines [57, 62]. Polyamines (spermine, spermidine, putrescine) are low molecular weight molecules that participate in cellular proliferation and DNA synthesis. They are known to interact with DNA bases and phosphate groups, and these adducts produce strong vibrations at the $1080-1100 \mathrm{~cm}^{-1}$ spectral domain [63]. Interestingly, the amount of excreted polyamines in urine increases after platinum-based chemotherapy in ovarian cancer, but this effect is mostly observed in patients with chemo-sensitive disease [64]. Therefore, the height of the band at $1080-1100 \mathrm{~cm}^{-1}$ may prove useful for monitoring response to chemotherapy, potentially through detection of complexes between polyamines and phosphate on cell-free DNA in urine. Of note, acetylated spermine has been investigated as a possible urinary biomarker for the diagnosis of ovarian cancer [65].

Contrary to what was observed in plasma and serum, absorbance at $1740 \mathrm{~cm}^{-1}$ wavelength (produced as previously stated by bonds in lipids) was stronger in the nonchemotherapy ovarian cancer group ( $\mathrm{RC} 0.5$ ) compared to NACT patients (RC 0.1) (Fig. 4). The effect of chemotherapy in urinary lipid concentrations has not been investigated, although a study has reported significantly lower phospholipids in urine of breast cancer patients following surgery (i.e., after reduction or elimination of tumour load) [66]. Therefore, more intense absorbances at $1740 \mathrm{~cm}^{-1}$ wavelength in blood biofluids post-NACT, with an opposite trend in urine featuring higher peaks before treatment initiation, might in combination suggest increased chemosensitivity.

An interesting outcome of urine spectroscopy was the presence of a marginal statistically significant difference $(P=$ 0.01 ) between patients with endometriosis and other benign gynaecological abnormalities (Fig. 1). This difference was not present in plasma and serum, suggesting a potential for ATRFTIR spectroscopy to classify endometriosis in urine samples. Previous studies have revealed some urinary biomarkers (mainly enzymes and peptides) with good discriminatory ability, using methods that included mass spectrometry but not vibrational spectroscopy $[67,68]$. However, further chemometric evaluation of this finding was outside the context of this study.

Two previous studies have investigated the performance of biofluids in ovarian cancer diagnosis with ATR-FTIR spectroscopy [18, 27]. In both studies, patients had not received chemotherapy. Gajjar et al. found classification accuracies of $96.67 \%$ for plasma and $95 \%$ for serum in a cohort comprising 30 ovarian cancer patients and 30 controls with benign gynaecological pathologies. Sensitivities and specificities were not calculated and, although it was stated that $86.7 \%$ of ovarian cancer patients had raised CA125 levels, CA125 concentrations were not reported; CA125 levels were not available for the benign control group. In their cohort, $60 \%$ of patients had early-stage disease (FIGO I and II) compared to
$46.5 \%$ in our study for the non-chemotherapy group, with similar distribution of ovarian cancer histological subtypes [18]. Paraskevaidi et al. assessed the performance of urine in a cohort comprising 10 ovarian cancer patients and 10 healthy controls (without stating if benign pathology was present) and found sensitivity, specificity and accuracy of up to $100 \%$, $97.5 \%$ and $98.3 \%$ respectively. CA125 levels were not available and, although it was reported that all ovarian cancer patients had high-grade disease, staging was not defined [27]. The differences in our outcomes (which were slightly lower for plasma and serum but markedly lower for urine) are probably due to the much bigger size of our cohort, which increased patient heterogeneity, but at the same time provides a more pragmatic estimate of ATR-FTIR spectrochemical performance towards ovarian cancer detection in the general population. This is particularly relevant for the study by Paraskevaidi et al., whose cohort size was approximately 20 times smaller than ours and might have compared advanced-stage ovarian cancer patients to individuals with absent pathology, thus optimising outcomes but at the same time not reflecting a "real-life" situation. The possibility of differences in technical parameters (such as methods of sample collection, preparation and spectroscopic measurements) between the two aforementioned studies and ours was ruled out, as same processes were used. It is also not possible to comment on whether epidemiological factors (such as age, BMI, comorbidities) have affected outcomes, as none of these studies (including ours) performed regression analyses for these variables.

In their study, Paraskevaidi et al. also identified potential diagnostic biomarkers, whereas biomarker assignment for the dataset included in the paper by Gajjar et al. was performed in a separate study by Owens et al. [28]. The latter identified intense absorbances in the ovarian cancer group at 1000 $1200 \mathrm{~cm}^{-1}$ wavelength area for plasma but not for serum. This is in partial agreement with our findings and reinforces our impression that circulating glycoproteins in blood (predominantly CA125) are strong classifiers of ovarian cancer detected through ATR-FTIR spectroscopy. With regard to urine, there was a single common peak (at $1040 \mathrm{~cm}^{-1}$ ) between our study and the one by Paraskevaidi et al., which as previously stated has been assigned to mucins [50]. Taken together, these observations suggest a consistency of spectroscopic changes that could be attributed to CA125 between different studies, highlighting its importance as a potential biomarker in detection of ovarian cancer through vibrational spectroscopy.

Several systematic reviews have investigated the performance of serum biomarkers in identification of ovarian malignancies. The CA125 assay exhibits $79 \%$ sensitivity and $78 \%$ specificity, whereas for HE4, sensitivities and specificities range between 74-79\% and 87-93\%, respectively [69, 70]. In our study, CA125 had similar rates with the ones reported 
in the literature, featuring $85 \%$ sensitivity and $75 \%$ specificity at the $35 \mathrm{u} / \mathrm{ml}$ cut-off threshold. The combined use of CA125 and HE4 can raise sensitivity up to $87 \%$ but specificity does not overcome $82 \%$ [70]. Predictive models that use pelvic ultrasonography alone or in combination with CA125 levels have demonstrated sensitivities up to $93 \%$ and specificities up to $92 \%$ [71]. However, these algorithms rely on the presence of a pelvic mass, which is not always present in ovarian cancer cases. In our study, ATR-FTIR spectroscopy of blood serum achieved $76 \%$ sensitivity (overall similar to serum biomarkers) but $98 \%$ specificity, which is superior to all the aforementioned modalities, and had excellent diagnostic accuracy (94\%). Compared to serum assays, it is more costeffective and quicker, as it does not require the use of reagents and produces results within minutes (instead of hours or occasionally days). In relation to ultrasonography, it is not operator dependent and can be associated with higher patient acceptance, as it avoids the discomfort and intimacy of transvaginal scanning. Additionally, it does not require the presence of a distinct ovarian tumour, which is a prerequisite in ultrasound-based models, making it applicable to any patient presenting with suspicious symptoms for ovarian cancer. Based on its high specificity, a negative test could reliably exclude the presence of ovarian cancer whereas a positive test would prompt further investigations, thus reducing the amount of unnecessary interventions and patient anxiety.

The main strengths of this study are the size of its cohort (the biggest employed so far in ATR-FTIR spectroscopy of biofluids for ovarian cancer detection) and its prospective design and consecutive recruitment of participants. In this field, the performance of blood-derived biofluids and urine is assessed simultaneously for the first time. It included patients with a wide range of benign gynaecological conditions as well as a variety of ovarian cancer histological subtypes and stages, providing a realistic approach to the encounter of these entities in the general population. It also involves the first attempt to detect chemotherapy-induced spectrochemical changes in biofluids of ovarian cancer patients, and a possible correlation of these alterations with prediction of chemosensitivity. Its main weaknesses are the lack of regression analyses to evaluate the impact of confounding factors, along with the lack of subgroup analyses to estimate performance in early-stagedisease detection, which is the main challenge in the timely diagnosis of ovarian cancer. These parameters would limit direct clinical application of blood serum ATR-FTIR spectroscopy, as a diagnostic tool in ovarian malignancy. Additionally, the assessment of chemotherapy effects was not conducted on a unique ovarian cancer patient group followed up linearly pre- and post-NACT, and as such, the reported outcomes should be regarded as preliminary indirect evidence about the potential of vibrational spectroscopy in investigating treatment response.
In conclusion, our study has shown that ATR-FTIR spectroscopy of blood-derived biofluids (predominantly serum) compares well with currently used diagnostic modalities in ovarian cancer, whereas urine demonstrated poor results. Spectrochemical alterations that can be attributed to circulating CA125 concentration and structural changes can serve as classifiers from benign gynaecological conditions and assessors of chemotherapy effects. Further research, ideally in a single patient cohort with sample collection before and after NACT, is required to validate these results and investigate correlations with tumour resectability at IDS as well as survival outcomes. Finally, future studies should address whether centrifugation can improve the performance of urine in spectroscopic diagnosis of ovarian cancer and determine its potential for classification of endometriosis through ATR-FTIR spectroscopy.

Supplementary Information The online version contains supplementary material available at https://doi.org/10.1007/s00216-021-03472-8.

Acknowledgements The authors thank the patients and staff of Royal Preston Hospital for facilitating these studies, with special thanks to their research technician, Katherine M. Ashton.

Data citations (1) Giamougiannis, P.; Martin-Hirsch, P.L.; Martin, F.L. 2021. Figshare. https://doi.org/10.6084/m9.figshare.14372024

Funding Funding from Rosemere Cancer Foundation at Royal Preston Hospital is gratefully acknowledged.

\section{Declarations}

Ethics approval and consent to participate This research has involved human participants that gave informed consent for providing samples, and has received full ethical approval by recognised bodies.

Conflict of interest FLM holds positions and shareholdings in Biocel UK Ltd. and its subsidiary companies; these companies are developing spectrochemical tests for commercial gain.

All other authors have no conflicts of interest to declare.

Open Access This article is licensed under a Creative Commons Attribution 4.0 International License, which permits use, sharing, adaptation, distribution and reproduction in any medium or format, as long as you give appropriate credit to the original author(s) and the source, provide a link to the Creative Commons licence, and indicate if changes were made. The images or other third party material in this article are included in the article's Creative Commons licence, unless indicated otherwise in a credit line to the material. If material is not included in the article's Creative Commons licence and your intended use is not permitted by statutory regulation or exceeds the permitted use, you will need to obtain permission directly from the copyright holder. To view a copy of this licence, visit http://creativecommons.org/licenses/by/4.0/. 


\section{References}

1. Bray F, Ferlay J, Soerjomataram I, Siegel RL, Torre LA, Jemal A. Global cancer statistics 2018: GLOBOCAN estimates of incidence and mortality worldwide for 36 cancers in 185 countries. CA Cancer J Clin. 2018;68(6):394-424. https://doi.org/10.3322/caac. 21492.

2. Reid F. The world ovarian cancer coalition atlas. Int Agency Res Cancer. 2018.

3. Stewart C, Ralyea C, Lockwood S. Ovarian cancer: an integrated review. Semin Oncol Nurs. 2019;35(2):151-6. https://doi.org/10. 1016/j.soncn.2019.02.001.

4. Matulonis UA, Sood AK, Fallowfield L, Howitt BE, Sehouli J, Karlan BY. Ovarian cancer. Nat Rev Dis Primers. 2016;2:16061. https://doi.org/10.1038/nrdp.2016.61.

5. Lheureux S, Gourley C, Vergote I, Oza AM. Epithelial ovarian cancer. Lancet. 2019;393(10177):1240-53. https://doi.org/10. 1016/S0140-6736(18)32552-2.

6. Jacobs IJ, Menon U, Ryan A, Gentry-Maharaj A, Burnell M, Kalsi $\mathrm{JK}$, et al. Ovarian cancer screening and mortality in the UK Collaborative Trial of Ovarian Cancer Screening (UKCTOCS): a randomised controlled trial. Lancet. 2016;387(10022):945-56. https://doi.org/10.1016/S0140-6736(15)01224-6.

7. Buys SS, Partridge E, Black A, Johnson CC, Lamerato L, Isaacs C, et al. Effect of screening on ovarian cancer mortality: the Prostate, Lung, Colorectal and Ovarian (PLCO) Cancer Screening Randomized Controlled Trial. JAMA. 2011;305(22):2295-303. https://oi.org/10.1001/jama.2011.766.

8. Jacobs I, Oram D, Fairbanks J, Turner J, Frost C, Grudzinskas JG. A risk of malignancy index incorporating CA 125, ultrasound and menopausal status for the accurate preoperative diagnosis of ovarian cancer. Br J Obstet Gynaecol. 1990;97(10):922-9. https://doi. org/10.1111/j.1471-0528.1990.tb02448.x.

9. Moore RG, McMeekin DS, Brown AK, DiSilvestro P, Miller MC, Allard WJ, et al. A novel multiple marker bioassay utilizing HE4 and CA125 for the prediction of ovarian cancer in patients with a pelvic mass. Gynecol Oncol. 2009;112(1):40-6. https://doi.org/10. 1016/j.ygyno.2008.08.031.

10. Aithal A, Rauth S, Kshirsagar P, Shah A, Lakshmanan I, Junker WM, et al. MUC16 as a novel target for cancer therapy. Expert Opin Ther Targets. 2018;22(8):675-86. https://doi.org/10.1080/ 14728222.2018.1498845.

11. Meden H, Fattahi-Meibodi A. CA 125 in benign gynecological conditions. Int J Biol Markers. 1998;13(4):231-7.

12. Karimi-Zarchi M, Dehshiri-Zadeh N, Sekhavat L, Nosouhi F. Correlation of CA-125 serum level and clinico-pathological characteristic of patients with endometriosis. Int J Reprod Biomed. 2016;14(11):713-8

13. Byrne HJ, Baranska M, Puppels GJ, Stone N, Wood B, Gough KM, et al. Spectropathology for the next generation: quo vadis? Analyst. 2015;140(7):2066-73. https://doi.org/10.1039/c4an02036g.

14. Baker MJ, Trevisan J, Bassan P, Bhargava R, Butler HJ, Dorling $\mathrm{KM}$, et al. Using Fourier transform IR spectroscopy to analyze biological materials. Nat Protoc. 2014;9:1771-91. https://doi.org/ 10.1038/nprot.2014.110.

15. Baker MJ, Hussain SR, Lovergne L, Untereiner V, Hughes C, Lukaszewski RA, et al. Developing and understanding biofluid vibrational spectroscopy: a critical review. Chem Soc Rev. 2016;45:1803-18. https://doi.org/10.1039/c5cs00585j.

16. Glassford SE, Byrne B, Kazarian SG. Recent applications of ATR FTIR spectroscopy and imaging to proteins. Biochim Biophys Acta. 2013;1834(12):2849-58. https://doi.org/10.1016/j.bbapap. 2013.07.015.

17. Martin FL, Kelly JG, Llabjani V, Martin-Hirsch PL, Patel II, Trevisan J, et al. Distinguishing cell types or populations based on the computational analysis of their infrared spectra. Nat Protoc. 2010;5(11):1748-60. https://doi.org/10.1038/nprot.2010. 133.

18. Gajjar K, Trevisan J, Owens G, Keating PJ, Wood NJ, Stringfellow $\mathrm{HF}$, et al. Fourier-transform infrared spectroscopy coupled with a classification machine for the analysis of blood plasma or serum: a novel diagnostic approach for ovarian cancer. Analyst. 2013;138(14):3917-26. https://doi.org/10.1039/c3an36654e.

19. Sitnikova VE, Kotkova MA, Nosenko TN, Kotkova TN, Martynova DM, Uspenskaya MV. Breast cancer detection by ATR-FTIR spectroscopy of blood serum and multivariate dataanalysis. Talanta. 2020;214:120857. https://doi.org/10.1016/j. talanta.2020.120857.

20. Butler HJ, Brennan PM, Cameron JM, Finlayson D, Hegarty MG, Jenkinson MD, et al. Development of high-throughput ATR-FTIR technology for rapid triage of brain cancer. Nat Commun. 2019;10(1):4501. https://doi.org/10.1038/s41467-019-12527-5.

21. Hands JR, Dorling KM, Abel P, Ashton KM, Brodbelt A, Davis C, et al. Attenuated total reflection Fourier transform infrared (ATRFTIR) spectral discrimination of brain tumour severity from serum samples. J Biophotonics. 2014;7(3-4):189-99. https://doi.org/10. 1002/jbio.201300149.

22. Medipally DKR, Cullen D, Untereiner V, Sockalingum GD, Maguire A, Nguyen TNQ, et al. Vibrational spectroscopy of liquid biopsies for prostate cancer diagnosis. Ther Adv Med Oncol. 2020;12:1758835920918499. https://doi.org/10.1177/ 1758835920918499 .

23. Maitra I, Morais CLM, Lima KMG, Ashton KM, Date RS, Martin FL. Attenuated total reflection Fourier-transform infrared spectral discrimination in human bodily fluids of oesophageal transformation to adenocarcinoma. Analyst. 2019;144(24):7447-56. https:// doi.org/10.1039/c9an01749f.

24. Habartová L, Bunganič $\mathrm{B}$, Tatarkovič M, Zavoral M, Vondroušová J, Syslová K, et al. Chiroptical spectroscopy and metabolomics for blood-based sensing of pancreatic cancer. Chirality. 2018;30(5): 581-91. https://doi.org/10.1002/chir.22834.

25. Tatarkovič M, Miškovičová M, Štovíčková L, Synytsya A, Petruželka L, Setnička V. The potential of chiroptical and vibrational spectroscopy of blood plasma for the discrimination between colon cancer patients and the control group. Analyst. 2015;140(7): 2287-93. https://doi.org/10.1039/c4an01880j.

26. Paraskevaidi M, Morais CLM, Ashton KM, Stringfellow HF, McVey RJ, Ryan NAJ, et al. Detecting endometrial cancer by blood spectroscopy: a diagnostic cross-sectional study. Cancers (Basel). 2020;12(5). https://doi.org/10.3390/cancers12051256.

27. Paraskevaidi M, Morais CLM, Lima KMG, Ashton KM, Stringfellow HF, Martin-Hirsch PL, et al. Potential of midinfrared spectroscopy as a non-invasive diagnostic test in urine for endometrial or ovarian cancer. Analyst. 2018;143(13):315663. https://doi.org/10.1039/c8an00027a.

28. Owens GL, Gajjar K, Trevisan J, Fogarty SW, Taylor SE, Da Gama-Rose B, et al. Vibrational biospectroscopy coupled with multivariate analysis extracts potentially diagnostic features in blood plasma/serum of ovarian cancer patients. J Biophotonics. 2014;7:200-9. https://doi.org/10.1002/jbio.201300157.

29. Martin FL, German MJ, Wit E, Fearn T, Ragavan N, Pollock HM. Identifying variables responsible for clustering in discriminant analysis of data from infrared microspectroscopy of a biological sample. J Comput Biol. 2007;14(9):1176-84. https://doi.org/10.1089/cmb. 2007.0057.

30. Trevisan J, Angelov PP, Carmichael PL, Scott AD, Martin FL. Extracting biological information with computational analysis of Fourier-transform infrared (FTIR) biospectroscopy datasets: current practices to future perspectives. Analyst. 2012;137(14):3202-15. https://doi.org/10.1039/c2an16300d. 
31. Zondervan KT, Becker CM, Koga K, Missmer SA, Taylor RN, Viganò P. Endometriosis. Nat Rev Dis Primers. 2018;4(1):9. https://doi.org/10.1038/s41572-018-0008-5.

32. Prat J. Staging classification for cancer of the ovary, fallopian tube, and peritoneum. Int J Gynaecol Obstet. 2014;124(1):1-5. https:// doi.org/10.1016/j.ijgo.2013.10.001.

33. Giamougiannis P, Morais CLM, Grabowska R, Ashton KM, Wood NJ, Martin-Hirsch PL, et al. A comparative analysis of different biofluids towards ovarian cancer diagnosis using Raman microspectroscopy. Anal Bioanal Chem. 2021;413(3):911-22. https://doi.org/10.1007/s00216-020-03045-1.

34. Morais CLM, Paraskevaidi M, Cui L, Fullwood NJ, Isabelle M, Lima KMG, et al. Standardization of complex biologically derived spectrochemical datasets. Nat Protoc. 2019;14(5):1546-77. https:// doi.org/10.1038/s41596-019-0150-x.

35. Bro R, Smilde AK. Principal component analysis. Anal Methods. 2014;6(9):2812-31.

36. Ballabio D, Consonni V. Classification tools in chemistry. Part 1: linear models. PLS-DA. Anal Methods. 2013;5(16):3790-8.

37. Hibbert DB Vocabulary of concepts and terms in chemometrics (IUPAC Recommendations 2016). 2016;88(4):407.

38. Brereton RG, Lloyd GR. Partial least squares discriminant analysis: taking the magic away. J Chemom. 2014;28(4):213-25.

39. Morais CLM, Santos MCD, Lima KMG, Martin FL. Improving data splitting for classification applications in spectrochemical analyses employing a random-mutation Kennard-Stone algorithm approach. Bioinformatics. 2019;35(24):5257-63. https://doi.org/10. 1093/bioinformatics/btz421.

40. Morais CLM, Lima KMG. Comparing unfolded and twodimensional discriminant analysis and support vector machines for classification of EEM data. Chemom Intell Lab Syst. 2017;170:1-12.

41. Wei JJ, William J, Bulun S. Endometriosis and ovarian cancer: a review of clinical, pathologic, and molecular aspects. Int J Gynecol Pathol. 2011;30(6):553-68. https://doi.org/10.1097/PGP. 0b013e31821f4b85.

42. Khajehpour M, Dashnau JL, Vanderkooi JM. Infrared spectroscopy used to evaluate glycosylation of proteins. Anal Biochem. 2006;348(1):40-8. https://doi.org/10.1016/j.ab.2005.10.009.

43. Derenne A, Derfoufi KM, Cowper B, Delporte C, Goormaghtigh E. FTIR spectroscopy as an analytical tool to compare glycosylation in therapeutic monoclonal antibodies. Anal Chim Acta. 2020;1112: 62-71. https://doi.org/10.1016/j.aca.2020.03.038.

44. Su KY, Lee WL. Fourier transform infrared spectroscopy as a cancer screening and diagnostic tool: a review and prospects. Cancers (Basel). 2020;12(1). https://doi.org/10.3390/cancers12010115.

45. Kirwan A, Utratna M, O'Dwyer ME, Joshi L, Kilcoyne M. Glycosylation-based serum biomarkers for cancer diagnostics and prognostics. Biomed Res Int. 2015;2015:490531. https://doi.org/ $10.1155 / 2015 / 490531$.

46. Hattrup CL, Gendler SJ. Structure and function of the cell surface (tethered) mucins. Annu Rev Physiol. 2008;70:431-57. https://doi. org/10.1146/annurev.physiol.70.113006.100659.

47. Moore RG, Brown AK, Miller MC, Skates S, Allard WJ, Verch T, et al. The use of multiple novel tumor biomarkers for the detection of ovarian carcinoma in patients with a pelvic mass. Gynecol Oncol. 2008;108(2):402-8. https://doi.org/10.1016/j.ygyno.2007. 10.017 .

48. Bian J, Li B, Kou XJ, Liu TZ, Ming L. Clinical significance of combined detection of serum tumor markers in diagnosis of patients with ovarian cancer. Asian Pac J Cancer Prev. 2013;14(11):6241-3. https://doi.org/10.7314/apjcp.2013.14.11.6241.
49. Haridas D, Ponnusamy MP, Chugh S, Lakshmanan I, Seshacharyulu P, Batra SK. MUC16: molecular analysis and its functional implications in benign and malignant conditions. FASEB J. 2014;28(10):4183-99. https://doi.org/10.1096/fj.14257352.

50. Lewis PD, Lewis KE, Ghosal R, Bayliss S, Lloyd AJ, Wills J, et al. Evaluation of FTIR spectroscopy as a diagnostic tool for lung cancer using sputum. BMC Cancer. 2010;10:640. https://doi.org/10. 1186/1471-2407-10-640.

51. Gill DJ, Clausen H, Bard F. Location, location, location: new insights into O-GalNAc protein glycosylation. Trends Cell Biol. 2011;21(3):149-58. https://doi.org/10.1016/j.tcb.2010.11.004.

52. Giamougiannis $\mathrm{P}$, Martin-Hirsch PL, Martin FL. The evolving role of MUC16 (CA125) in the transformation of ovarian cells and the progression of neoplasia. Carcinogenesis. 2021 Apr 17;42(3):32743. https://doi.org/10.1093/carcin/bgab010.

53. Davis HM, Zurawski VR Jr, Bast RC Jr, Klug TL. Characterization of the CA 125 antigen associated with human epithelial ovarian carcinomas. Cancer Res. 1986;46(12 Pt 1):6143-8.

54. Saldova R, Wormald MR, Dwek RA, Rudd PM. Glycosylation changes on serum glycoproteins in ovarian cancer may contribute to disease pathogenesis. Dis Markers. 2008;25(4-5):219-32. https://doi.org/10.1155/2008/601583.

55. Wiercigroch E, Szafraniec E, Czamara K, Pacia MZ, Majzner K, Kochan K, et al. Raman and infrared spectroscopy of carbohydrates: a review. Spectrochim Acta A Mol Biomol Spectrosc. 2017;185:317-35. https://doi.org/10.1016/j.saa.2017.05.045.

56. Kovács A, Nyerges B, Izvekov V. Vibrational analysis of N-acetylalpha-D-glucosamine and beta-D-glucuronic acid. J Phys Chem B. 2008;112(18):5728-35. https://doi.org/10.1021/jp710432d.

57. Movasaghi Z, Rehman S, Ur Rehman DI. Fourier transform infrared (FTIR) spectroscopy of biological tissues. Appl Spectrosc Rev. 2008;43(2):134-79.

58. Willemse PM, van der Meer RW, Burggraaf J, van Elderen SG, de Kam ML, de Roos A, et al. Abdominal visceral and subcutaneous fat increase, insulin resistance and hyperlipidemia in testicular cancer patients treated with cisplatin-based chemotherapy. Acta Oncol. 2014;53(3):351-60. https://doi.org/10.3109/0284186X.2013. 819116.

59. Tian W, Yao Y, Fan G, Zhou Y, Wu M, Xu D, et al. Changes in lipid profiles during and after (neo)adjuvant chemotherapy in women with early-stage breast cancer: a retrospective study. PLoS One. 2019;14(8):e0221866. https://doi.org/10.1371/journal.pone. 0221866.

60. Zhao G, Cardenas H, Matei D. Ovarian cancer-why lipids matter. Cancers (Basel). 2019;11(12). https://doi.org/10.3390/ cancers 11121870 .

61. Tay SK, Chua EK. Correlation of serum, urinary and salivary CA 125 levels in patients with adnexal masses. Ann Acad Med Singap. 1994;23(3):311-4.

62. Bertoluzza A, Fagnano C, Finelli P, Morelli MA, Simoni R, Tosi R. Raman and infrared spectra of spermidine and spermine and their hydrochlorides and phosphates as a basis for the study of the interactions between polyamines and nucleic acids. J Raman Spectrosc. 1983;14(6):386-94.

63. Ouameur AA, Tajmir-Riahi HA. Structural analysis of DNA interactions with biogenic polyamines and cobalt(III)hexamine studied by Fourier transform infrared and capillary electrophoresis. J Biol Chem. 2004;279(40):42041-54. https://doi.org/10.1074/jbc. M406053200.

64. Lawton FG, Griffin M, Slack JA, Blackledge G. Predicting response to chemotherapy for patients with epithelial ovarian cancer 
using urinary polyamine excretion patterns. Br J Cancer. 1990;62(4):692-4. https://doi.org/10.1038/bjc.1990.359.

65. Niemi RJ, Roine AN, Häkkinen MR, Kumpulainen PS, Keinänen TA, Vepsäläinen JJ, et al. Urinary polyamines as biomarkers for ovarian cancer. Int J Gynecol Cancer. 2017;27(7):1360-6. https:// doi.org/10.1097/IGC.0000000000001031.

66. Kim H, Min HK, Kong G, Moon MH. Quantitative analysis of phosphatidylcholines and phosphatidylethanolamines in urine of patients with breast cancer by nanoflow liquid chromatography/ tandem mass spectrometry. Anal Bioanal Chem. 2009;393(6-7): 1649-56. https://doi.org/10.1007/s00216-009-2621-3.

67. Fassbender A, Burney RO, Occc DF, D'Hooghe T, Giudice L. Update on biomarkers for the detection of endometriosis. Biomed Res Int. 2015;2015:130854. https://doi.org/10.1155/2015/130854.

68. Wang L, Liu HY, Shi HH, Lang JH, Sun W. Urine peptide patterns for non-invasive diagnosis of endometriosis: a preliminary prospective study. Eur J Obstet Gynecol Reprod Biol. 2014;177:23-8. https://doi.org/10.1016/j.ejogrb.2014.03.011.
69. Ferraro S, Braga F, Lanzoni M, Boracchi P, Biganzoli EM, Panteghini M. Serum human epididymis protein 4 vs carbohydrate antigen 125 for ovarian cancer diagnosis: a systematic review. J Clin Pathol. 2013;66(4):273-81. https://doi.org/10.1136/jclinpath2012-201031.

70. Lin J, Qin J, Sangvatanakul V. Human epididymis protein 4 for differential diagnosis between benign gynecologic disease and ovarian cancer: a systematic review and meta-analysis. Eur J Obstet Gynecol Reprod Biol. 2013;167(1):81-5. https://doi.org/ 10.1016/j.ejogrb.2012.10.036.

71. Meys EM, Kaijser J, Kruitwagen RF, Slangen BF, Van Calster B, Aertgeerts B, et al. Subjective assessment versus ultrasound models to diagnose ovarian cancer: a systematic review and meta-analysis. Eur J Cancer. 2016;58:17-29. https://doi.org/10.1016/j.ejca.2016. 01.007.

Publisher's note Springer Nature remains neutral with regard to jurisdictional claims in published maps and institutional affiliations. 